\title{
Isolation of biosynthesis related transcripts of 2,3,5,4'-tetrahydroxy stilbene-2-O- $\beta$-D-glucoside from Fallopia multiflora by suppression subtractive hybridization
}

\author{
Wei Zhao ${ }^{1,2}$, Shujing Sheng ${ }^{2}$, Zhongyu Liu², Di Lu², Kuanpeng Zhu ${ }^{1,2}$, Xiaoze Li ${ }^{1,2}$, Shujin Zhao ${ }^{2 *}$, Yan Yao ${ }^{3}$ \\ ${ }^{1} S$ chool of Bioscience and Bioengineering, South China University of Technology, Guangzhou 510006, People's Republic of China \\ 2 Department of Pharmacy, General Hospital of Guangzhou Military Command, Guangzhou 510010, People's Republic of China \\ ${ }^{3}$ School of Life Sciences, Guangzhou University, Guangzhou 510006, People's Republic of China
}

\section{Abstract}

2,3,5,4'-tetrahydroxy stilbene-2-O-B-D-glucoside (THSG) exerts multiple pharmacodynamic actions, found in Fallopia multiflora, but the biosynthesis pathway of THSG is still unclear. To clear this ambiguity, we constructed suppression subtractive hybridization (SSH) libraries to screen the genes involved in THSG biosynthesis from two F. multiflora varieties, which vary significantly in THSG content. Twelve non-redundant differentially expressed sequence tags were obtained and the full lengths of 4 unreported fragments were amplified by rapid amplification of cDNA ends. We totally got 7 fulllength transcripts, and all of them were aligned to the transcriptome and digital gene expression tag profiling database of four F. multiflora tissues (root, stem and leaf from Deqing F. multiflora and another root from Chongqing F. multiflora; data unpublished) using local BLAST. The results showed that there was a significant, organ specific difference in the expression of fragments and full-length sequences. All the sequences were annotated by aligning to nucleotide and protein databases. Kyoto Encyclopedia of Genes and Genomes pathway analysis indicated that THSG biosynthesis was correlated with multiple life activities.

Keywords: Fallopia multiflora; gene expression difference; transcriptome; $2,3,5,4$ '-tetrahydroxy stilbene-2-O- $\beta$-Dglucooside

\section{Introduction}

Fallopia multiflora is a traditional Chinese medicinal herb, which has been widely used for thousands of years. Research showed that F. multiflora has potent antioxidative and cytoprotective properties [1], enhanced purgative effects, promoted diuresis and choleretic effects [2], and exerted a neuroprotective effect against glutamate-induced neurotoxicity [3]. 2,3,5,4'-tetrahydroxy stilbene-2-O-B-Dglucoside (THSG) is an active component in F. multiflora, which possesses anti-hyperlipidemic [4], anti-oxidative, anti-inflammatory, endothelial-protective activities [5]. Furthermore, THSG can protect osteoblastic MC3T3-E1 cells via inhibiting the release of bone-resorbing mediators and oxidative damage of the cells [6], and suppress atherosclerosis by altering the expression of key proteins that may be novel molecular targets responsible for atherogenesis [7]. Sun et al. who reported that THSG might provide a potentially new strategy for preventing and treating neurodegenerative

\footnotetext{
*Corresponding author. Email: gzzsjzhs@163.com
}

Handling Editor: Przemysław Wojtaszek disorders such as Parkinson's disease, also showed that THSG may protect neurons against MPP+- induced cell death through improving mitochondrial function, decreasing oxidative stress and inhibiting apoptosis [8]. The pharmacological research revealed that THSG has a potential impact on human health. As a stilbene, THSG and resveratrol (3,5,4'-trihydroxy-trans-stilbene) belong to phenylpropanoids characterized by a 1,2-diphenylethylene backbone. Plant stilbenes are derived from phenylalanine biosynthesis via the general phenylpropanoid pathway. One p-coumaroylCoA or cinnamoyl-CoA derived from the phenylpropanoid pathway is ligated with three malonyl-CoA under the catalysis of stilbene synthase (STS) and stilbene is the product [9]. STS is a polyketide synthase (PKS) belonging to type III PKSs. Other PKSs III include resveratrol synthase (RS), chalcone synthases (CHS), bibenzyl synthase (BBS), stilbene carboxylate synthase (STCS), 4-coumaroyltriacetate lactone synthase (CTAS) and more [10]. Every PKSs III catalyzes the synthesis of one unique stilbene. Up to now, no enzyme was found to catalyze the biosynthesis of THSG. Sheng et al. isolated a stilbene synthase gene FmPKS from the rhizomes of $F$. multiflora. The gene expression pattern in the plant correlated with the THSG content in different tissues, but 
THSG was still not detectable in transgenic Arabidopsis thaliana in which FmPKS was inserted and expressed [11]. Shao et al. showed precursor feeding of methyl jasmonate and salicylic acid in suspension cultures of F. multiflora that could increase THSG production [12]. It is still not clear if THSG is synthesized via phenylalanine pathway or some other way. Suppression subtractive hybridization (SSH) is helpful in identifying differentially expressed genes. Extensive studies showed that $\mathrm{SSH}$ is a powerful tool in the analysis of stress resistance [13], pathological mechanism [14] and developmental physiology [15]. Moreover, Wang et al. found that light could be effective for activation of the biosynthesis of phenylpropanoids by establishing SSH cDNA libraries of tea calli [16]. In this study, we attempted to identify the genes that are related to THSG biosynthesis by SSH. Full-length sequences were obtained using $3^{\prime} 5{ }^{\prime} \mathrm{RACE}$, and then the gene functions were annotated by blastn to nucleotide databases $\mathrm{nt}$, and blastx to protein databases nr, Swiss-Prot, Kyoto Encyclopedia of Genes and Genomes (KEGG) and clusters of orthologous groups (COG). Meanwhile, comparative analysis with the transcriptome and digital expression profile revealed the expression differences for each gene in various tissues of F. multiflora. This may indicate that these genes are associated with THSG synthesis.

\section{Material and methods}

\section{Plant material collection}

F. multiflora plants were gathered from 13 cities (counties) in March 2010, which includes Guangxi Province (Nanning City, Guilin City, Jingxi County, Tianlin County), Guangdong Province (Guangzhou City, Shenzhen City, Dongguan City, Deqing City, Zhaoqing City, Gaozhou City, Zhanjiang City), Jiangxi Province (Xinyu City), Chongqing City. As some samples were gathered in the field, the age was unclear. All plant materials were maintained in the medicinal plant garden of the Department of Pharmacy, Guangzhou Liuhuaqiao Hospital, Guangzhou, China.

\section{HPLC analysis of THSG}

To quantify the THSG in F. multiflora roots, fresh plant materials were frozen in liquid nitrogen and ground to fine powder in a mortar. After vacuum freeze-drying, $0.2 \mathrm{~g}$ of each sample was taken up in $25 \mathrm{ml} 50 \%(\mathrm{v} / \mathrm{v})$ methanol and then refluxed at room temperature for $16 \mathrm{~h}$. After being filtered through a $0.22 \mu \mathrm{m}$ film, $10 \mu \mathrm{l}$ of filtrate was analyzed by HPLC using a Dikma Diamonsil C18 column $(250 \times 4.6 \mathrm{~mm}$, tablets path $5 \mu \mathrm{m})$. Chromatographic separation was performed using a solvent system of $\mathrm{H}_{2} \mathrm{O}$ and $\mathrm{CH}_{3} \mathrm{CN}$ with the ratio of $3: 1(\mathrm{v} / \mathrm{v})$ over $10 \mathrm{~min}$. The flow rate was $1 \mathrm{ml} / \mathrm{min}$, with detection at $320 \mathrm{~nm}$. Each data point represents the average of three independent experiments.

\section{Total RNA extraction and mRNA purification}

Total RNA was extracted from leaves and roots using the Plant Total RNA Isolation kit (Bioteke, China) and treated with DNase I (TaKaRa, Dalian, China). Ethidium bromide (EtBr) staining, agarose gel electrophoresis and spectrophotometric (NanoDrop 2000, USA) analysis were performed to examine the quality and concentration of total RNA. mRNA was purified using Oligo te ${ }^{\mathrm{Tm}}-\mathrm{dT}^{30}<$ SUPER $>$ mRNA Purification Kit (from Total RNA) according to the manufacturer's instructions (TaKaRa, Dalian, China).

\section{Suppression subtractive hybridization (SSH)}

Driver and tester cDNA was synthesized from two samples at equal amounts of purified mRNA, using SMARTer PCR cDNA Synthesis K it (Clontech, USA) according to the manufacturer's instructions. After repeated twice hybridization, target genes were amplified using nested PCR by Advantage $^{\mathrm{mm}} \mathrm{cDNA}$ PCR Kit (Clontech, USA). To raise the PCR efficiency, an adapter was added to the nested PCR primers (Tab. 1; homo nested primer). The following target genes were amplified using Fermentas Dream Taq (Fermentas, USA) with homo PCR primer (Tab. 1; homo PCR primer).

The PCR products were inserted into the PMD19-T vector (TaKaRa, Dalian, China), and the ligated products were transformed into Escherichia coli DH5a competent cells by heat shock. Then plated onto LB medium containing $100 \mu \mathrm{g} /$ $\mathrm{ml}$ ampicillin, $24 \mu \mathrm{g} / \mathrm{ml} \mathrm{IPTG}$ and $20 \mu \mathrm{g} / \mathrm{ml} \mathrm{X}$-gal, and incubated overnight at $37^{\circ} \mathrm{C}$. Recombinant white colonies were randomly selected for colony PCR with universal primers (Tab. 1; universal primers). Colonies containing cloned fragments were sent for sequencing. Besides control sample (Poly $\mathrm{A}^{+}$RNA from human skeletal muscle) provided with the kit, which was used as a control driver cDNA, the experiments were performed with 3 different testers: I - cDNA from Guilin's root sample as tester and Chongqing's root sample as driver; II - Chongqing's root sample as tester and Guilin's root sample as driver; III - Guilin's leaf sample as tester and Guilin's root sample as driver.

\section{Cloning genes with full length by RACE}

The $3^{\prime} 5^{\prime} \mathrm{RACE}$ of the candidate genes was performed in purified mRNA using PCR-select ${ }^{\mathrm{Tm}} \mathrm{cDNA}$ subtraction kit (Clontech, USA) according to the manufacturer's instruction. To improve the specificity, nested primers were designed for Nested-PCR besides gene specific primers. Fragments of 4 genes were selected to do $3^{\prime} 5^{\prime} \mathrm{RACE}$ from SSH library after BLAST in NCBI (Tab. 1). The Nested-PCR reaction was performed by Advantage cDNA Polymerase Mix (Clontech, USA) with the following thermal cycling parameters: $94^{\circ} \mathrm{C}$ for $4 \mathrm{~min}$, followed by 35 cycles of $94^{\circ} \mathrm{C}$ for $30 \mathrm{~s}, 68^{\circ} \mathrm{C}$ for $30 \mathrm{~s}$ and $72^{\circ} \mathrm{C}$ for $1.5 \mathrm{~min}$, and the final extension was performed at $72^{\circ} \mathrm{C}$ for $10 \mathrm{~min}$. The PCR product was cloned into the pMD19-T vector (TaKaRa, Dalian, China) and sequenced.

\section{Sequence analysis}

Gene fragments obtained from SSH and RACE were compared with the sequences in nucleotide collection database and expressed sequence tag (EST) database at NCBI using the BLASTN algorithm. Further annotation was carried out using nr, Swiss-Prot, gene ontology database (GO), COG and KEGG. Clustalx1.83 and Contig Express software were used for multiple sequence alignment, sequence identity and linkage. Moreover the obtained sequences were compared with the transcriptome and digital gene expression tag profiling (DGE) database of four different tissue samples (Cr: roots of Chongqing F. multiflora; Dr: roots of Deqing 
Tab. 1 SSH primers and RACE primers of 4 selected gene fragments.

\begin{tabular}{|c|c|c|}
\hline Gene & Primer & Sequence \\
\hline & Homo nested primer & 5'-GCGACCTACAACATGGCTACCGTCGAGCGGCCGCCCGGGCAGGT \\
\hline & homo PCR primer & 5'-GCGACCTACAACATGGCTACCG \\
\hline & universal primer & 5'-GAGCGGATAACAATTTCACACAGG \\
\hline \multirow[t]{4}{*}{ GE } & $3^{\prime} \mathrm{GSP}$ & 5'-CTGGCAACTGTTGAAGGACGCGAAGA \\
\hline & $5^{\prime}$ GSP & 5'-GCCCAAGCACCAAATGCCTCTGC \\
\hline & $3^{\prime}$ Nested primer & 5'-AGAGAGGTAGCAACAGATGGAGG \\
\hline & $5^{\prime}$ Nested primer & 5'-ССТCСАTCTGTTGCTAССТСТCT \\
\hline \multirow[t]{4}{*}{$G W$} & $3^{\prime} \mathrm{GSP}$ & 5'-GTCTCATGCGCTCCTCCTCCAGCC \\
\hline & $5^{\prime}$ GSP & 5'-GGCGAGCAGAGAAGCAAAGGCTGG \\
\hline & $3^{\prime}$ Nested primer & 5'-AGCCTTTGCTTCTCTGCTCGCCT \\
\hline & $5^{\prime}$ Nested primer & 5'-GGAGGAGCGCATGAGACAGAACA \\
\hline \multirow[t]{4}{*}{$C F$} & $3^{\prime}$ GSP & 5'-AGGCAATGGAGAAAAGCCGCTCGC \\
\hline & $5^{\prime} \mathrm{GSP}$ & 5'-TAGGCAACGGCAATCCCAGCACGG \\
\hline & $3^{\prime}$ Nested primer & 5'-GGATTGCCGTTGCCTAAAGTGTG \\
\hline & $5^{\prime}$ Nested primer & 5'-TTGCTCATTCСТАСАСТССТCGC \\
\hline \multirow[t]{4}{*}{$C A$} & $3^{\prime} \mathrm{GSP}$ & 5'-CTTGGGCTTTTTCAGGGACAGACG \\
\hline & $5^{\prime} \mathrm{GSP}$ & 5'-CCGGTAGAGGCCATCAGGATGCAG \\
\hline & $3^{\prime}$ Nested primer & 5'-CAGACGAAAAGACGAGAATGAGG \\
\hline & $5^{\prime}$ Nested primer & 5'-TTCCTCTTTGGCTTTCCСАCTTC \\
\hline
\end{tabular}

"G" represents the origin of the sample, Guilin city; "C" represents Chongqing city.

F. multiflora; Dl: leaves of Deqing F. multiflora; Ds: stems of Deqing F. multiflora) of F. multiflora (unpublished data) using local BLAST.

\section{Results}

\section{Quantification of THSG}

To select the specific samples with robust difference in THSG concentration, HPLC was used to analyze the THSG contents. Results indicated that there was a significant difference $(P$-value $<0.001)$ in THSG content in F. multiflora root from different origins (Fig. 1, Tab. 2). Root samples from Guilin City and Zhaoqin City have the highest accumulation of THSG, with a content of $6.37 \%$ and $5.25 \%$ respectively. Chongqing samples have the lowest THSG level, with a content of $0.001 \%$. Significant differences were found even within Guilin samples. For example, Guilin samples, the lowest is $1.46 \%$, while the highest is $6.37 \%$. According to this data, we choose Guilin and Chongqing samples as the following materials.

\section{SSH fragments comparison analysis}

SSH fragments were amplified and cloned into pMD19$\mathrm{T}$ vector. A total of 136 colonies were picked up, and the insertions were confirmed by PCR using universal primers. 75 clones among the 136 colonies were positive (55.1\%) and sequenced, with insertion length ranging from 0.2 to $0.5 \mathrm{~kb}$ (data not shown). Removing redundant sequences, 12 fragments were obtained, and they were compared with the sequences in nucleotide and EST database at NCBI using the BLASTN algorithm. Results are shown in Tab. 3. There are
4 sequences with no significant similarity in the databases. The 4 sequences were all obtained from the roots and named GE, GW, CF, CA for subsequent research ("G" represent the origin of the sample, Guilin city; "C" represent Chongqing city.). All sequences were submitted to the NCBI GenBank (accession Nos. JZ469200 to JZ469209) for public domain use, except YCTS4 and GTS2 (ribosomal RNA).

Then the 12 sequences were compared with the transcriptome and DEG database of F. multiflora (unpublished data) using local BLAST. Significantly similar genes identified in the transcriptome database were initially aligned by blastx to protein databases nr, Swiss-Prot, KEGG and COG (evalue $<0.00001)$, and then aligned by blastn to nucleotide database nt $(e$-value $<0.00001)$. Proteins with the highest sequence similarity were retrieved with the given Unigenes and their functional annotations (Tab. 4).

\section{3'5'RACE and full-length sequences comparison analysis}

The full-length sequences of GE, GW, CF and CA were cloned by $3^{\prime} \mathrm{RACE}$ and 5'RACE. More than one full-length sequence was obtained for all genes but one, CF. All sequences have been submitted to the NCBI GenBank (accession Nos. KF054163 to KF054169) for public domain. Meanwhile the sequences were compared with the transcriptome and DEG database using local BLAST. Significantly similar Unigenes in transcriptome were aligned by blastx to protein databases, and aligned by blastn to nucleotide databases nt to annotate gene function (Tab. 5).

\section{Pathway annotation}

Different genes generally interact with each other to sustain their biological functions. Pathway-based analysis 


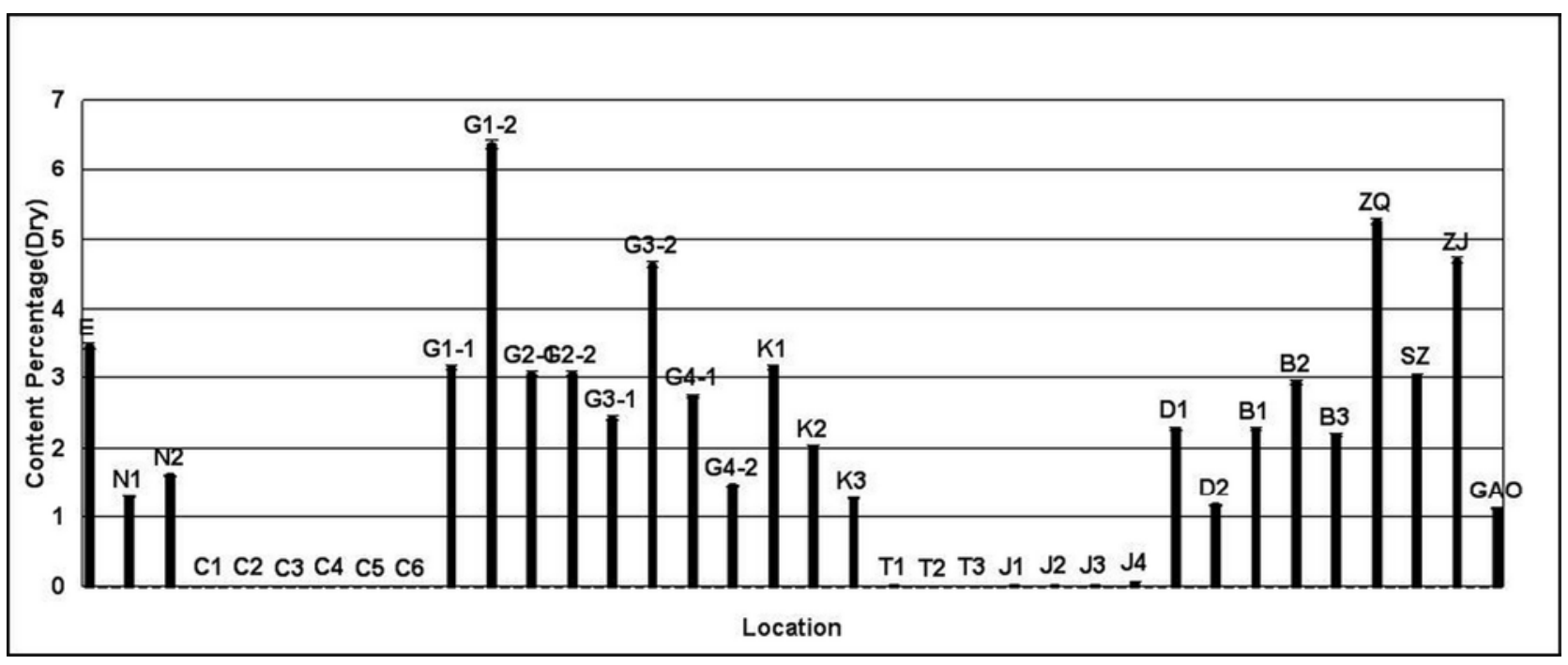

Fig. 1 THSG content in F. multiflora plants from different areas. Content percentage (dry): (THSG quality/quality of $F$. multiflora powder $) \times 100$. All samples are the roots of $F$. multiflora, except Gl, Gs, Dl and Ds. B - Guangzhou City; C - Chongqing City; D - Deqing City; E - Dongguan City; G - Guilin City; GAO - Gaozhou City; J - Xinyu City; K - Jingxi County; N - Nanning City; SZ - Shenzhen City; T - Tianlin County; ZJ - Zhanjiang City; ZQ - Zhaoqing City. Dl - leaves of Deqing F. multiflora; Ds: stems of Deqing F. multiflora; Gl - leaves of Guilin F. multiflora; Gs: stems of Guilin F. multiflora.

Tab. 2 Quantification of THSG in F. multiflora plants from different areas $(n=3)$.

\begin{tabular}{|c|c|c|c|c|c|c|c|}
\hline Sample & $\begin{array}{c}\text { Average } \\
\text { concentration }(\mathrm{ng} / \mu \mathrm{l})\end{array}$ & $\begin{array}{c}\text { Average } \\
\text { concentration (\%) }\end{array}$ & $\begin{array}{l}\text { Standard } \\
\text { deviation }\end{array}$ & Sample & $\begin{array}{c}\text { Average } \\
\text { concentration }(\mathrm{ng} / \mu \mathrm{l})\end{array}$ & $\begin{array}{c}\text { Average } \\
\text { concentration (\%) }\end{array}$ & $\begin{array}{l}\text { Standard } \\
\text { deviation }\end{array}$ \\
\hline E & 272.68 & 3.35 & 0.4487 & K1 & 252.88 & 3.15 & 0.0125 \\
\hline $\mathrm{N} 1$ & 103.36 & 1.29 & 0.0105 & K2 & 162.80 & 2.01 & 0.0091 \\
\hline $\mathrm{N} 2$ & 128.56 & 1.61 & 0.0004 & K3 & 101.32 & 1.26 & 0.0009 \\
\hline $\mathrm{C} 1$ & 0.79 & 0.01 & 0.0004 & $\mathrm{~T} 1$ & 2.08 & 0.03 & 0.0001 \\
\hline $\mathrm{C} 2$ & 1.08 & 0.01 & 0.0002 & $\mathrm{~T} 2$ & 0.28 & 0.00 & 0.0002 \\
\hline C3 & 0.08 & 0.00 & 0.0000 & $\mathrm{~T} 3$ & 1.32 & 0.02 & 0.0069 \\
\hline $\mathrm{C} 4$ & 0.88 & 0.01 & 0.0001 & $\mathrm{~J} 1$ & 2.24 & 0.03 & 0.0005 \\
\hline C5 & 0.60 & 0.01 & 0.0002 & $\mathrm{~J} 2$ & 3.08 & 0.04 & 0.0001 \\
\hline C6 & 0.36 & 0.00 & 0.0006 & $\mathrm{~J} 3$ & 1.36 & 0.03 & 0.0007 \\
\hline G1-1 & 253.52 & 3.15 & 0.0116 & $\mathrm{~J} 4$ & 6.40 & 0.08 & 0.0013 \\
\hline G1-2 & 509.96 & 6.37 & 0.0582 & D1 & 185.68 & 2.27 & 0.0244 \\
\hline G2-1 & 244.96 & 3.05 & 0.0127 & $\mathrm{D} 2$ & 96.72 & 1.16 & 0.0104 \\
\hline G2-2 & 254.00 & 3.06 & 0.0086 & $\mathrm{~B} 1$ & 182.36 & 2.26 & 0.0758 \\
\hline G3-1 & 194.08 & 2.43 & 0.0006 & B2 & 245.04 & 2.94 & 0.0995 \\
\hline G3-2 & 372.20 & 4.64 & 0.0179 & B3 & 148.08 & 2.18 & 0.0534 \\
\hline G4-1 & 221.28 & 2.73 & 0.0135 & ZQ & 230.04 & 5.25 & 0.0411 \\
\hline G4-2 & 117.64 & 1.46 & 0.0039 & ZJ & 390.50 & 4.70 & 0.0572 \\
\hline SZ & 248.25 & 3.03 & 0.0092 & GAO & 93.00 & 1.12 & 0.0121 \\
\hline $\mathrm{Gl}$ & 3.22 & 0.04 & 0.0007 & $\mathrm{Dl}$ & 1.56 & 0.02 & 0.0105 \\
\hline Gs & 84.36 & 1.01 & 0.0120 & Ds & 45.91 & 0.58 & 0.0057 \\
\hline
\end{tabular}

Concentration \%: (THSG quality/quality of F. multiflora powder) $\times 100$. All samples are the roots of $F$. multiflora, except Gl, Gs, Dl and Ds. B - Guangzhou City; C - Chongqing City; D - Deqing City; E - Dongguan City; G - Guilin City; GAO - Gaozhou City; J - Xinyu City; K - Jingxi County; N - Nanning City; SZ - Shenzhen City; T - Tianlin County; ZJ - Zhanjiang City; ZQ - Zhaoqing City. Dl - leaves of Deqing F. multiflora; Ds: stems of Deqing F. multiflora; Gl - leaves of Guilin F. multiflora; Gs: stems of Guilin F. multiflora. 
Tab. 3 Parameters of high similarity SSH sequence alignments, identified by BLASTN in nucleotide and EST NCBI databases.

\begin{tabular}{|c|c|c|c|c|c|}
\hline Gene & Description & Query cover & $e$-value & Max ident. & Accession \\
\hline YCTS1 & Rheum australe catalase mRNA & $27 \%$ & $4 e-35$ & $94 \%$ & EU931220.1 \\
\hline \multirow[t]{3}{*}{ YCTS2 } & Salicornia europaea TUB mRNA for alpha tubulin, partial cds & $95 \%$ & $2 e-71$ & $90 \%$ & AB437373.1 \\
\hline & Panicum virgatum clone PV_ABa073-K05, complete sequence & $96 \%$ & $2 \mathrm{e}-70$ & $90 \%$ & AC243247.1 \\
\hline & Phyllostachys edulis cDNA clone: bphylf024i19, full insert sequence & $96 \%$ & $2 \mathrm{e}-70$ & $90 \%$ & FP093842.1 \\
\hline YCTS3 & $\begin{array}{l}\text { Mesembryanthemum crystallinum major latex protein homolog mRNA, } \\
\text { complete cds }\end{array}$ & $44 \%$ & $4 e-29$ & $85 \%$ & AF054445.1 \\
\hline YCTS4 & Sesbania drummondii clone SSH-47_02_F03_T7, mRNA sequence & $80 \%$ & $5 e-56$ & $95 \%$ & DQ465800.1 \\
\hline \multirow[t]{4}{*}{ GTS1 } & $\begin{array}{l}\text { LEAF460 Polygonum sibiricum leaf Knorringia sibirica cDNA clone LEAF460, } \\
\text { mRNA sequence }\end{array}$ & $48 \%$ & $8 e-40$ & $93 \%$ & FE903382.1 \\
\hline & $\begin{array}{l}\text { XHBC-B22 Anas platyrhynchos muscle-related, library constructed by SSH } \\
\text { Anas platyrhynchos }\end{array}$ & $28 \%$ & $2 e-26$ & $99 \%$ & HS410797.1 \\
\hline & $\begin{array}{l}\text { cDNA, mRNA sequence, leaf_cn } 90 \text { cDNA library from leaf of Polygonum } \\
\text { minus }\end{array}$ & $49 \%$ & $2 \mathrm{e}-30$ & $87 \%$ & JG745134.1 \\
\hline & Persicaria minor cDNA clone leaf_cn90, mRNA sequence & & & & \\
\hline GTS2 & Fallopia multiflora voucher DB1 $18 \mathrm{~S}$ ribosomal RNA, gene, complete sequence & $85 \%$ & $9 e-58$ & $98 \%$ & EF153706.1 \\
\hline YTS1 & Brassica rapa subsp. pekinensis thionin mRNA, complete cds & $36 \%$ & $4 e-09$ & $79 \%$ & AF090836.1 \\
\hline CTS1 & Rheum australe catalase mRNA, complete cds & $46 \%$ & $2 e-35$ & $94 \%$ & EU931220.1 \\
\hline
\end{tabular}

The character before "TS" represent the tester; "YCTS" means the fragment was found from both "Y" and "C" testers. C - roots of Chongqing F. multiflora; G - roots of Guilin F. multiflora; Y - leaves of Guilin F. multiflora.

Tab. 4 Parameters and annotation of high similarity SSH sequence alignments, identified by local BLAST in F. multiflora transcriptome databases.

\begin{tabular}{|c|c|c|c|c|c|c|c|}
\hline Gene & Unigene & Score & $e$-value & Gene length & Nt-annotation & Nr-annotation & Accession \\
\hline \multirow[t]{2}{*}{$G E$} & CL3180.Contig2_TrCD & 244 & $2 e-64$ & 1125 & $\begin{array}{l}\text { Vitis vinifera clone } \\
\text { SS0AEB22YD16 }\end{array}$ & $\begin{array}{l}\text { PREDICTED: vacuolar } \\
\text { protein-sorting-associated } \\
\text { protein } 37 \text { homolog } 2 \text { (Vitis } \\
\text { vinifera) }\end{array}$ & FQ388706 \\
\hline & CL3180.Contig1_TrCD & 244 & $2 e-64$ & 1234 & $\begin{array}{l}\text { Vitis vinifera clone } \\
\text { SS0AEB22YD16 }\end{array}$ & $\begin{array}{l}\text { PREDICTED: vacuolar } \\
\text { protein-sorting-associated } \\
\text { protein } 37 \text { homolog } 2 \text { (Vitis } \\
\text { vinifera) }\end{array}$ & FQ388706 \\
\hline \multirow[t]{2}{*}{$G W$} & CL5835.Contig2_TrCD & 254 & $2 e-67$ & 1520 & $\begin{array}{l}\text { PREDICTED: Vitis vinifera } \\
\text { GPN-loop GTPase } 1 \text { homolog- } \\
\text { like (LOC100249973), mRNA }\end{array}$ & $\begin{array}{l}\text { hypothetical protein } \\
\text { VITISV_029075 (Vitis } \\
\text { vinifera) }\end{array}$ & XM_002279556 \\
\hline & CL5835.Contig1_TrCD & 254 & $2 e-67$ & 1523 & $\begin{array}{l}\text { PREDICTED: Vitis vinifera } \\
\text { GPN-loop GTPase } 1 \text { homolog- } \\
\text { like (LOC100249973), mRNA }\end{array}$ & $\begin{array}{l}\text { hypothetical protein } \\
\text { VITISV_029075 (Vitis } \\
\text { vinifera) }\end{array}$ & XM_002279556 \\
\hline \multirow[t]{3}{*}{$C F$} & CL9534.Contig2_TrCD & 480 & e-135 & 1155 & $\begin{array}{l}\text { PREDICTED: Glycine } \\
\text { max uncharacterized } \\
\text { protein LOC100797178 } \\
\text { (LOC100797178), mRNA }\end{array}$ & $\begin{array}{l}\text { predicted protein (Populus } \\
\text { trichocarpa) }\end{array}$ & XM_003517340 \\
\hline & CL9534.Contig1_TrCD & 480 & e-135 & 1168 & $\begin{array}{l}\text { PREDICTED: Glycine } \\
\text { max uncharacterized } \\
\text { protein LOC100797178 } \\
\text { (LOC100797178), mRNA }\end{array}$ & $\begin{array}{l}\text { predicted protein (Populus } \\
\text { trichocarpa) }\end{array}$ & XM_003517340 \\
\hline & CL8316.Contig2_TrCD & 480 & e-135 & 1137 & $\begin{array}{l}\text { PREDICTED: Glycine } \\
\text { max uncharacterized } \\
\text { protein LOC100797178 } \\
\text { (LOC100797178), mRNA }\end{array}$ & $\begin{array}{l}\text { predicted protein (Populus } \\
\text { trichocarpa) }\end{array}$ & XM_003517340 \\
\hline
\end{tabular}


Tab. 4 (continued)

\begin{tabular}{|c|c|c|c|c|c|c|c|}
\hline Gene & Unigene & Score & $e$-value & Gene length & Nt-annotation & Nr-annotation & Accession \\
\hline & CL8316.Contig1_TrCD & 480 & e-135 & 1150 & $\begin{array}{l}\text { PREDICTED: Glycine } \\
\text { max uncharacterized } \\
\text { protein LOC100797178 } \\
\text { (LOC100797178), mRNA }\end{array}$ & $\begin{array}{l}\text { predicted protein (Populus } \\
\text { trichocarpa) }\end{array}$ & XM_003517340 \\
\hline$C A$ & Unigene22710_TrCD & 418 & $\mathrm{e}-117$ & 1174 & $\begin{array}{l}\text { PREDICTED: Vitis } \\
\text { vinifera uncharacterized } \\
\text { LOC100248615 } \\
\text { (LOC100248615), mRNA }\end{array}$ & $\begin{array}{l}\text { PREDICTED: uncharacterized } \\
\text { protein } \\
\text { LOC100248615 (Vitis vinifera) }\end{array}$ & XM_002274928 \\
\hline \multirow[t]{2}{*}{ YCTS1 } & CL3542.Contig3_TrCD & 234 & $5 e-61$ & 2031 & $\begin{array}{l}\text { Rheum australe catalase } \\
\text { mRNA, complete cds }\end{array}$ & catalase (Rheum australe) & EU931220 \\
\hline & CL3542.Contig1_TrCD & 111 & $5 e-24$ & 487 & $\begin{array}{l}\text { Rheum australe catalase } \\
\text { mRNA, complete cds }\end{array}$ & catalase (Rheum australe) & EU931220 \\
\hline \multirow[t]{3}{*}{ YCTS2 } & CL2077.Contig2_TrCD & 434 & $\mathrm{e}-121$ & 1663 & $\begin{array}{l}\text { P. amygdalus mRNA for alpha- } \\
\text { tubulin }\end{array}$ & $\begin{array}{l}\text { tubulin alpha chain, putative } \\
\text { (Ricinus communis) }\end{array}$ & $\mathrm{X} 67162$ \\
\hline & CL2077.Contig1_TrCD & 111 & $3 e-24$ & 564 & $\begin{array}{l}\text { Populus trichocarpa tubulin } \\
\text { alpha-8 chain (TUA8), mRNA }\end{array}$ & $\begin{array}{l}\text { alpha-tubulin (Gossypium } \\
\text { hirsutum) }\end{array}$ & EF151304 \\
\hline & CL2077.Contig7_TrCD & 96 & $2 \mathrm{e}-19$ & 394 & $\begin{array}{l}\text { PREDICTED: Vitis vinifera } \\
\text { tubulin alpha- } 3 \text { chain-like } \\
\text { (LOC100258974), mRNA }\end{array}$ & $\begin{array}{l}\text { alpha-tubulin (Miscanthus } \\
\text { sinensis) }\end{array}$ & XM_002281631 \\
\hline \multirow[t]{2}{*}{ YCTS3 } & CL5036.Contig1_TrCD & 521 & e-147 & 756 & $\begin{array}{l}\text { Mesembryanthemum } \\
\text { crystallinum major latex } \\
\text { protein homolog mRNA, } \\
\text { complete cds }\end{array}$ & $\begin{array}{l}\text { major latex protein homolog } \\
\text { (Mesembryanthemum } \\
\text { crystallinum) }\end{array}$ & AF054445 \\
\hline & CL5036.Contig2_TrCD & 210 & $5 e-54$ & 455 & - & $\begin{array}{l}\text { major latex protein homolog } \\
\text { (Mesembryanthemum } \\
\text { crystallinum) }\end{array}$ & AF054445 \\
\hline YCTS4 & CL8135.Contig1_TrCD & 236 & $5 e-62$ & 1672 & $\begin{array}{l}\text { Polygonum sachalinense } \\
26 \mathrm{~S} \text { ribosomal RNA gene, } \\
\text { complete sequence }\end{array}$ & $\begin{array}{l}\text { hypothetical protein } \\
\text { MTR_5g050970 (Medicago } \\
\text { truncatula) }\end{array}$ & AF479085 \\
\hline GTS1 & CL8017.Contig1_TrCD & 212 & $6 e-55$ & 834 & $\begin{array}{l}\text { Phyllostachys edulis cDNA } \\
\text { clone: bphyst } 022 \mathrm{a} 10 \text {, full insert } \\
\text { sequence }\end{array}$ & $\begin{array}{l}\text { Chain A, structure of Psbp } \\
\text { protein from Spinacia oleracea } \\
\text { at } 1.98 \text { A resolution }\end{array}$ & FP094014 \\
\hline GTS2 & CL10591.Contig1_TrCD & 248 & $e-65$ & 2806 & $\begin{array}{l}\text { Fallopia multiflora voucher } \\
\text { DX } 18 \text { S ribosomal RNA gene, } \\
\text { complete sequence }\end{array}$ & $\begin{array}{l}\text { Cytochrome P450 likeTBP } \\
\text { (Medicago truncatula) }\end{array}$ & EF153701 \\
\hline \multirow[t]{2}{*}{ CTS1 } & CL3542.Contig3_TrCD & 230 & $e-60$ & 2031 & $\begin{array}{l}\text { Rheum australe catalase } \\
\text { mRNA, complete cds }\end{array}$ & catalase (Rheum australe) & EU931220 \\
\hline & CL3542.Contig1_TrCD & 111 & $\mathrm{e}-24$ & 487 & $\begin{array}{l}\text { Rheum australe catalase } \\
\text { mRNA, complete cds }\end{array}$ & catalase (Rheum australe) & EU931220 \\
\hline \multirow[t]{4}{*}{ YTS1 } & CL5290.Contig2_TrCD & 486 & e-137 & 780 & $\begin{array}{l}\text { Brassica napus clone Bn } 3872 \\
\text { thionin precursor, mRNA, } \\
\text { complete cds }\end{array}$ & $\begin{array}{l}\text { crambin precursor = thionin } \\
\text { variant Thi2Ca10 (Crambe } \\
\text { abyssinica, seeds, Peptide } \\
\text { Partial, } 134 \text { aa) }\end{array}$ & EU887266 \\
\hline & CL5290.Contig1_TrCD & 486 & e-137 & 1094 & $\begin{array}{l}\text { Boechera divaricarpa isolate } \\
\text { SLW-C-C05 mRNA sequence }\end{array}$ & $\begin{array}{l}\text { crambin precursor = thionin } \\
\text { variant Thi2Ca10 (Crambe } \\
\text { abyssinica, seeds, Peptide } \\
\text { Partial, } 134 \text { aa) }\end{array}$ & DQ226844 \\
\hline & Unigene31799_TrCD & 113 & $9 e-25$ & 712 & $\begin{array}{l}\text { Boechera divaricarpa isolate } \\
\text { SLW-C-C05 mRNA sequence }\end{array}$ & $\begin{array}{l}\text { crambin precursor }=\text { thionin } \\
\text { variant Thi2Ca10 (Crambe } \\
\text { abyssinica, seeds, Peptide } \\
\text { Partial, } 134 \text { aa) }\end{array}$ & DQ226844 \\
\hline & CL5290.Contig3_TrCD & 113 & $9 e-25$ & 427 & $\begin{array}{l}\text { Brassica napus clone Bn } 3872 \\
\text { thionin precursor, mRNA, } \\
\text { complete cds }\end{array}$ & $\begin{array}{l}\text { thionin precursor (Brassica } \\
\text { napus) }\end{array}$ & EU887266 \\
\hline
\end{tabular}


Tab. 5 Parameters and annotation of high similarity full-length sequences alignments, identified by local BLAST in F. multiflora transcriptome databases.

\begin{tabular}{|c|c|c|c|c|c|c|c|}
\hline Gene & Unigene & Gene length & Similarity bp & $e$-value & Nt-annotation & Nr-annotation & Accession \\
\hline \multirow[t]{2}{*}{ GEfl } & CL3180.Contig2_TrCD & 1125 & 805 & 0 & $\begin{array}{l}\text { Vitis vinifera clone } \\
\text { SS0AEB22YD16 }\end{array}$ & $\begin{array}{l}\text { PREDICTED: vacuolar } \\
\text { protein-sorting-associated } \\
\text { protein } 37 \text { homolog } 2 \text { (Vitis } \\
\text { vinifera) }\end{array}$ & FQ388706 \\
\hline & CL3180.Contig1_TrCD & 1234 & 805 & 0 & $\begin{array}{l}\text { Vitis vinifera clone } \\
\text { SS0AEB22YD16 }\end{array}$ & $\begin{array}{l}\text { PREDICTED: vacuolar } \\
\text { protein-sorting-associated } \\
\text { protein } 37 \text { homolog } 2 \text { (Vitis } \\
\text { vinifera) }\end{array}$ & FQ388706 \\
\hline \multirow[t]{2}{*}{ GWfl1 } & Unigene6178_TrCD & 532 & 451 & 0 & $\begin{array}{l}\text { PREDICTED: Vitis vinifera } \\
\text { 40S ribosomal protein S30- } \\
\text { like (LOC100241142), } \\
\text { mRNA }\end{array}$ & $\begin{array}{l}\text { unnamed protein product } \\
\text { (Vitis vinifera) }\end{array}$ & XM_002281340 \\
\hline & Unigene20297_TrCD & 467 & 190 & $e-46$ & $\begin{array}{l}\text { PREDICTED: Vitis vinifera } \\
\text { 40S ribosomal protein S30- } \\
\text { like (LOC100252467), } \\
\text { mRNA }\end{array}$ & $\begin{array}{l}\text { unnamed protein product } \\
\text { (Vitis vinifera) }\end{array}$ & XM_002282997 \\
\hline GWfl2 & CL3625.Contig1_TrCD & 1188 & 436 & 0 & $\begin{array}{l}\text { PREDICTED: Vitis } \\
\text { vinifera katanin p } 80 \\
\text { WD40 repeat-containing } \\
\text { subunit B1 homolog 1-like } \\
\text { (LOC100247509), mRNA }\end{array}$ & $\begin{array}{l}\text { PREDICTED: katanin p80 } \\
\text { WD40 repeat-containing } \\
\text { subunit B1 homolog 1-like } \\
\text { (Glycine max) }\end{array}$ & XM_002264675 \\
\hline \multirow[t]{6}{*}{ GWfl3 } & CL3504.Contig1_TrCD & 868 & 664 & 0 & $\begin{array}{l}\text { Vigna radiata ubiquitin- } \\
\text { conjugating enzyme E2 } \\
\text { mRNA, complete cds }\end{array}$ & $\begin{array}{l}\text { PREDICTED: ubiquitin- } \\
\text { conjugating enzyme E2 10- } \\
\text { like (Glycine max) }\end{array}$ & FJ436357 \\
\hline & Unigene6181_TrCD & 979 & 445 & $e-62$ & $\begin{array}{l}\text { Camellia sinensis ubiquitin- } \\
\text { conjugating enzyme E2 } \\
\text { mRNA, complete cds }\end{array}$ & $\begin{array}{l}\text { PREDICTED: ubiquitin- } \\
\text { conjugating enzyme E2 10- } \\
\text { like (Glycine max) }\end{array}$ & JN400596 \\
\hline & CL3504.Contig3_TrCD & 973 & 443 & $4 e-47$ & $\begin{array}{l}\text { PREDICTED: Vitis vinifera } \\
\text { ubiquitin-conjugating } \\
\text { enzyme (LOC100232965), } \\
\text { mRNA }\end{array}$ & $\begin{array}{l}\text { predicted protein (Populus } \\
\text { trichocarpa) }\end{array}$ & XM_003633920 \\
\hline & CL3504.Contig4_TrCD & 915 & 405 & $7 e-46$ & $\begin{array}{l}\text { PREDICTED: Vitis vinifera } \\
\text { ubiquitin-conjugating } \\
\text { enzyme (LOC100232965), } \\
\text { mRNA }\end{array}$ & $\begin{array}{l}\text { ubiquitin-conjugating } \\
\text { enzyme E2-17 kDa } \\
\text { (Solanum lycopersicum) }\end{array}$ & XM_003633920 \\
\hline & Unigene27674_TrCD & 818 & 306 & $2 e-39$ & $\begin{array}{l}\text { PREDICTED: Vitis vinifera } \\
\text { ubiquitin-conjugating } \\
\text { enzyme (LOC100232965), } \\
\text { mRNA }\end{array}$ & $\begin{array}{l}\text { E2 ubiquitin-conjugating } \\
\text { enzyme UBC10 (Brassica } \\
\text { napus) }\end{array}$ & XM_003633920 \\
\hline & Unigene7679_TrCD & 520 & 243 & $5 e-16$ & $\begin{array}{l}\text { Solanum tuberosum } \\
\text { clone } 116 \mathrm{D} 11 \text { ubiquitin- } \\
\text { conjugating protein-like } \\
\text { mRNA, complete cds }\end{array}$ & $\begin{array}{l}\text { ubiquitin-conjugating } \\
\text { enzyme E2 } 28 \text { (Arabidopsis } \\
\text { thaliana) }\end{array}$ & DQ284472 \\
\hline \multirow[t]{3}{*}{$C F f l$} & CL9534.Contig2_TrCD & 1155 & 144 & $3 e-72$ & $\begin{array}{l}\text { PREDICTED: Glycine } \\
\text { max uncharacterized } \\
\text { protein LOC100797178 } \\
\text { (LOC100797178), mRNA }\end{array}$ & $\begin{array}{l}\text { predicted protein (Populus } \\
\text { trichocarpa) }\end{array}$ & XM_003517340 \\
\hline & CL9534.Contig1_TrCD & 1168 & 144 & $3 e-72$ & $\begin{array}{l}\text { PREDICTED: Glycine } \\
\text { max uncharacterized } \\
\text { protein LOC100797178 } \\
\text { (LOC100797178), mRNA }\end{array}$ & $\begin{array}{l}\text { predicted protein (Populus } \\
\text { trichocarpa) }\end{array}$ & XM_003517340 \\
\hline & CL8316.Contig2_TrCD & 1137 & 144 & $3 e-72$ & $\begin{array}{l}\text { PREDICTED: Glycine } \\
\text { max uncharacterized } \\
\text { protein LOC100797178 } \\
\text { (LOC100797178), mRNA }\end{array}$ & $\begin{array}{l}\text { predicted protein (Populus } \\
\text { trichocarpa) }\end{array}$ & XM_003517340 \\
\hline
\end{tabular}


Tab. 5 (continued)

\begin{tabular}{|c|c|c|c|c|c|c|c|}
\hline Gene & Unigene & Gene length & Similarity bp & $e$-value & Nt-annotation & Nr-annotation & Accession \\
\hline & CL8316.Contig1_TrCD & 1150 & 144 & $3 e-72$ & $\begin{array}{l}\text { PREDICTED: Glycine } \\
\text { max uncharacterized } \\
\text { protein LOC100797178 } \\
\text { (LOC100797178), mRNA }\end{array}$ & $\begin{array}{l}\text { predicted protein (Populus } \\
\text { trichocarpa) }\end{array}$ & XM_003517340 \\
\hline \multirow[t]{2}{*}{ CWfl1 } & Unigene22710_TrCD & 1174 & 316 & $3 e-158$ & $\begin{array}{l}\text { PREDICTED: Vitis } \\
\text { vinifera uncharacterized } \\
\text { LOC100248615 } \\
\text { (LOC100248615), mRNA }\end{array}$ & $\begin{array}{l}\text { PREDICTED: } \\
\text { uncharacterized protein } \\
\text { LOC100248615 (Vitis } \\
\text { vinifera) }\end{array}$ & XM_002274928 \\
\hline & CL4034.Contig3_TrCD & 2016 & 209 & $7 e-104$ & $\begin{array}{l}\text { PREDICTED: Vitis vinifera } \\
\text { pumilio homolog 1-like } \\
\text { (LOC100243338), mRNA }\end{array}$ & $\begin{array}{l}\text { PREDICTED: pumilio } \\
\text { homolog 1-like (Vitis } \\
\text { vinifera) }\end{array}$ & XM_002283155 \\
\hline \multirow[t]{2}{*}{ CWfl2 } & CL2293.Contig2_TrCD & 2481 & 165 & $9 e-82$ & $\begin{array}{l}\text { PREDICTED: Vitis } \\
\text { vinifera uncharacterized } \\
\text { LOC100246727 } \\
\text { (LOC100246727), mRNA }\end{array}$ & $\begin{array}{l}\text { expressed protein, putative } \\
\text { (Ricinus communis) }\end{array}$ & XM_002273173 \\
\hline & CL2293.Contig1_TrCD & 2572 & 165 & $9 e-82$ & $\begin{array}{l}\text { PREDICTED: Vitis } \\
\text { vinifera uncharacterized } \\
\text { LOC100246727 } \\
\text { (LOC100246727), mRNA }\end{array}$ & $\begin{array}{l}\text { expressed protein, putative } \\
\text { (Ricinus communis) }\end{array}$ & XM_002273173 \\
\hline
\end{tabular}

helps to further understand genes' biological functions. According to KEGG, the pathways of isolated genes are diverse in function. These genes were mainly involved in metabolic pathways; ubiquitin mediated proteolysis, pathogenic E. coli infection, amyotrophic lateral sclerosis (ALS), tryptophan metabolism, photosynthesis, gap junction, RIG-I-like receptor signaling pathway, mRNA surveillance pathway, peroxisome, endocytosis, ribosome, biosynthesis of secondary metabolites, phagosome, protein processing in endoplasmic reticulum, methane metabolism, glyoxylate and dicarboxylate metabolism, microbial metabolism in diverse environments, and RNA transport (Tab. 6).

\section{Comparison of genes expression and THSG content in different tissues}

Genes expression of SSH fragments in different plant tissues according to DGE database was shown in Fig. 2. Expression of GE, GW, CF and YTS1 in different plant tissues has no obvious difference. Gene expression of full- length sequences in different plant tissues has significant differences (Fig. 3), except GEfl and CFfl. Comparison between the expression of isolated genes and THSG content in different tissues revealed that the expression of GWfl1 (Unigene20297_TrCD), GWfl3 (Unigene27674) and CWfl1 (CL4034.Contig3_TrCD) is in parallel with THSG content in different tissues. Expression of CA, YCTS4 and CWfl1 (Unigene22710_TrCD) is contrary to THSG content in different tissues. Expression of $C A$, CTS1, GWfl2 (CL3625.Contig1_TrCD), GWfl3 (CL3504. Contig1_TrCD), YCTS4 and CWfl1 (Unigene22710_TrCD) in $\mathrm{Cr}$ are higher than in all $\mathrm{D}$ sample tissues.

\section{Discussion}

As of any secondary metabolite in plant, the production of THSG in F. multiflora is influenced by multiple factors, such as temperature, climate, altitude, precipitation etc. Gene screening between samples of distinct product content by SSH could display the mRNA information associated with the production of THSG. DGE profile of different plant tissues contains genes expression information in these tissues. Comparison with DGE profile showed the high level of activity of targeted genes in each tissue. The genes whose expression was significantly different were selected. Similar pattern of difference in gene abundance and THSG content in different tissues confirm that the gene might be associated with THSG biosynthesis.

In our study, the expression of GWfl1 (Unigene20297_ TrCD), GWfl3 (Unigene27674_TrCD) and CWfl1 (CL4034. Contig3_TrCD) is in parallel with THSG content in different tissues. Blast in NCBI database showed they might code $40 \mathrm{~S}$ ribosomal protein, ubiquitin-conjugating enzyme and RNA-binding protein of the Puf family. The predicted protein function indicated there should be active protein degradation and expression behavior in root sample. Expression of CA, YCTS4 and CWfl1 (Unigene22710_TrCD) is contrary to THSG content in different tissues. And CTS1 is higher expressed in Cr than Dr, Ds and Dl. They might be inhibitors of THSG biosynthesis. As roots always contain the highest amount of THSG, we consider the root is most likely the site of THSG synthesis. The fragments, which were obtained from Guilin root tester should be focused on. $G W$ (CL5835.Contig1_TrCD/CL5835.Contig2_TrCD) and GTS1 (CL8017.Contig1_TrCD) might be involved in providing energy for secondary metabolism via GTP metabolism and photosynthesis. GWfl2 (CL3625.Contig1_TrCD) is annotated as Katanin p80 WD40 repeat-containing subunit B1, GWfl3 (CL3504.Contig1_TrCD) as Ubiquitin-protein ligase. As these fragments were identified in SSH when Guilin root tester was applied, it means the intermediate process of THSG biosynthesis should be fast and efficient, and related protein is likely to be subjected to rapid degradation. GTS2 (CL10591. Contig1_TrCD) has high homology with Cytochrome P450 
Tab. 6 Pathway annotation of SSH sequences and full-length sequences participated.

\begin{tabular}{|c|c|c|c|}
\hline Gene & KOID & Definition & Pathway \\
\hline$G E$ & K12185 & VPS37; ESCRT-I complex subunit VPS37 & ko04144 endocytosis \\
\hline$G W$ & K06883 & & \\
\hline$C F$ & - & & \\
\hline$C A$ & - & & \\
\hline YCTS1 & K03781 & katE, CAT, catB, srpA; catalase (EC:1.11.1.6) & $\begin{array}{l}\text { ko01120 microbial metabolism in diverse environments; ko01100 } \\
\text { metabolic pathways; ko01110 biosynthesis of secondary metabolites; } \\
\text { ko00630 glyoxylate and dicarboxylate metabolism; ko00680 methane } \\
\text { metabolism; ko00380 tryptophan metabolism; ko04146 peroxisome; } \\
\text { ko05014 amyotrophic lateral sclerosis (ALS) }\end{array}$ \\
\hline YCTS2 & K07374 & TUBA; tubulin alpha & $\begin{array}{l}\text { ko04145 phagosome; ko04540 gap junction; ko05130 pathogenic } \\
\text { Escherichia coli infection }\end{array}$ \\
\hline YCTS3 & - & & \\
\hline YCTS4 & - & & \\
\hline GTS1 & K02717 & $\begin{array}{l}\text { psbP; photosystem II oxygen-evolving enhancer } \\
\text { protein } 2\end{array}$ & ko01100 metabolic pathways; ko00195 photosynthesis \\
\hline GTS2 & K14325 & $\begin{array}{l}\text { RNPS1; RNA-binding protein with serine-rich } \\
\text { domain } 1\end{array}$ & ko03013 RNA transport; ko03015 mRNA surveillance pathway \\
\hline CTS1 & K03781 & katE, CAT, catB, srpA; catalase (EC:1.11.1.6) & $\begin{array}{l}\text { ko01120 microbial metabolism in diverse environments; ko01100 } \\
\text { metabolic pathways; ko01110 biosynthesis of secondary metabolites; } \\
\text { ko00630 glyoxylate and dicarboxylate metabolism; ko00680 methane } \\
\text { metabolism; ko00380 tryptophan metabolism; ko04146 peroxisome; } \\
\text { ko05014 amyotrophic lateral sclerosis (ALS) }\end{array}$ \\
\hline YTS1 & - & & \\
\hline GEfl & K12185 & VPS37; ESCRT-I complex subunit VPS37 & ko04144 endocytosis \\
\hline GWfll & K02983 & $\begin{array}{l}\text { RP-S30e, RPS30; small subunit ribosomal protein } \\
\text { S30e }\end{array}$ & ko03010 ribosome \\
\hline GWfl2 & - & & \\
\hline GWfl3 & K06689 & $\begin{array}{l}\text { UBE2D_E, UBC4, UBC5; ubiquitin-conjugating } \\
\text { enzyme E2 D/E (EC:6.3.2.19) }\end{array}$ & $\begin{array}{l}\text { ko04141 protein processing in endoplasmic reticulum; ko04120 ubiquitin } \\
\text { mediated proteolysis }\end{array}$ \\
\hline CFfl & - & & \\
\hline CWfl1 & K14844 & $\begin{array}{l}\text { PUF6; pumilio homology domain family } \\
\text { member } 6\end{array}$ & ko03009 ribosome biogenesis \\
\hline CWfl2 & K12655 & $\begin{array}{l}\text { OTUD5, DUBA; OTU domain-containing } \\
\text { protein } 5 \text { (EC:3.1.2.15) }\end{array}$ & ko04622 RIG-I-like receptor signaling pathway \\
\hline
\end{tabular}

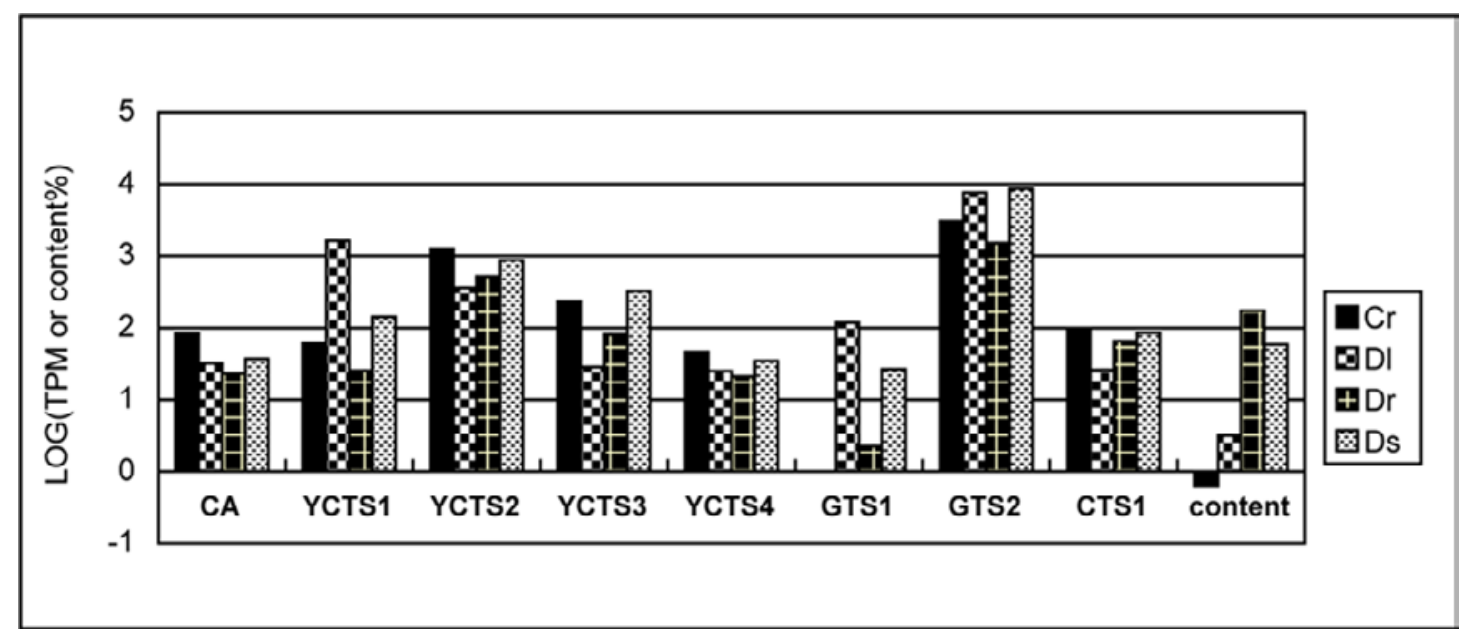

Fig. 2 Comparison of SSH fragments expression and THSG content in different tissues. Cr - roots of Chongqing F. multiflora; Dl - leaves of Deqing F. multiflora; Dr - roots of Deqing F. multiflora; Ds - stems of Deqing F. multiflora; TPM - transcripts per million clean tags (all data was calculated as the base of $\log 10$ ). 


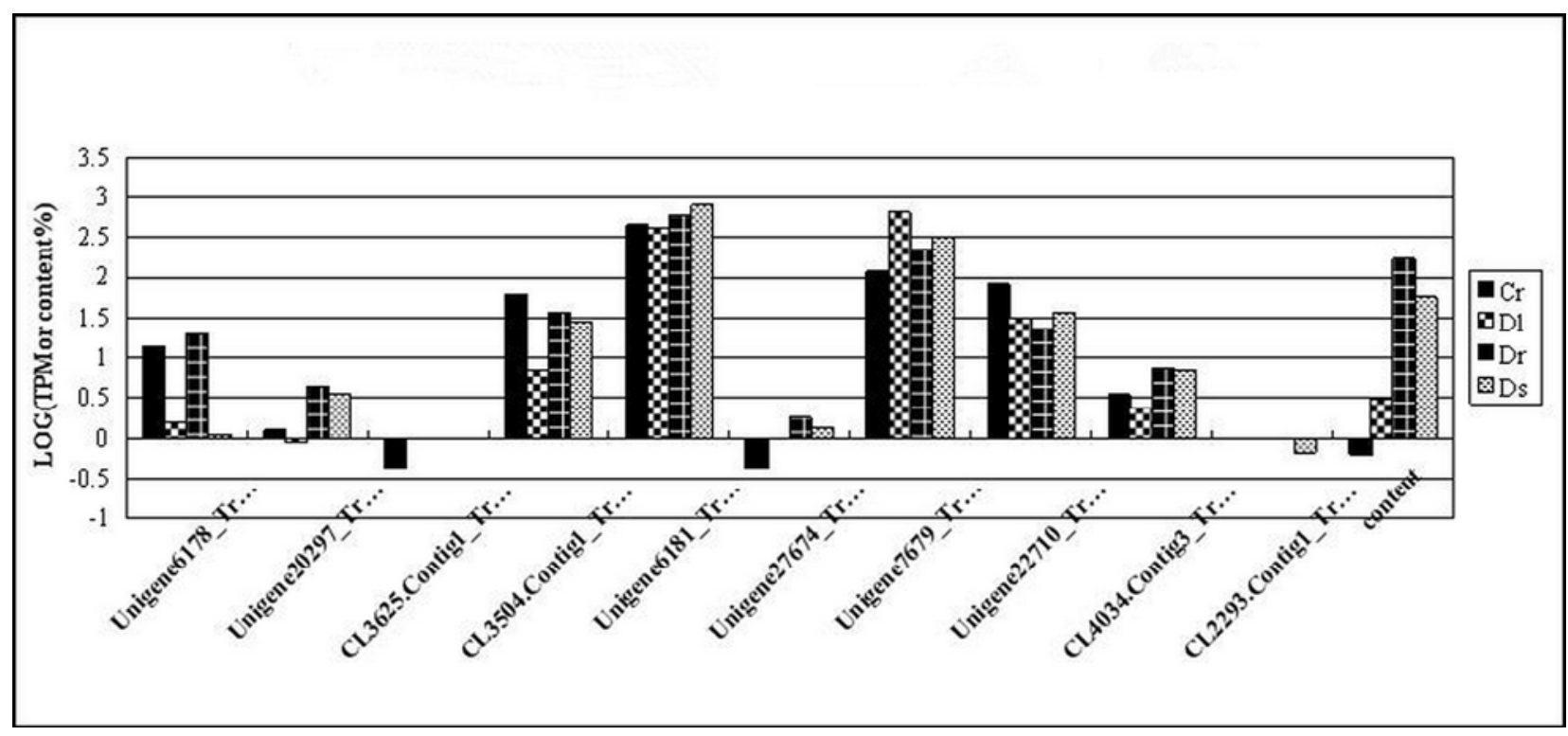

Fig. 3 Comparison of full-length sequences expression and THSG content in different tissues . Cr - roots of Chongqing F. multiflora; $\mathrm{Dl}$ - leaves of Deqing F. multiflora; Dr - roots of Deqing F. multiflora; Ds - stems of Deqing F. multiflora; TPM - transcripts per million clean tags (all data was calculated as the base of $\log 10$ ).

in Medicago truncatula. Cytochrome P450 was found be involved as multifunctional oxidases in the biosynthesis of many secondary metabolites like triterpenoid [17], glycyrrhizin [18], hemolytic saponins [19] and many more. Does it take part in THSG biosynthesis?

If THSG is derived from phenylalanine pathway with other stilbenes, then the CTS1 gene obtained from Chongqing root tester may be related with the inhibition of THSG biosynthesis. CTS1 gene is annotated as catalase and participates in "biosynthesis of secondary metabolites (ko01110)"; "glyoxylate and dicarboxylate metabolism (ko00630)" and

\section{Acknowledgments}

This work was funded by grants from Natural Science Foundation of Guangdong Province (10151001002000012), Science and Technology Department of Guangdong Province (2010B060200009).

\section{Authors' contributions}

The following declarations about authors' contributions to the research have been made: conceived and designed the experiments: WZ, SS, ZL; performed the experiments: WZ, DL, KZ, XL, YY; performed the analysis: WZ, SZ; wrote the paper: WZ; revised the paper: SZ, SS.

\section{References}

1. Steele ML, Truong J, Govindaraghavan S, Ooi L, Sucher NJ, Münch G. Cytoprotective properties of traditional Chinese medicinal herbal extracts in hydrogen peroxide challenged human U373 astroglia cells. Neurochem Int. 2013;62(5):522-529. http://dx.doi.org/10.1016/j. neuint.2012.08.018

2. Xie W, Zhao Y, Du L. Emerging approaches of traditional Chinese medicine formulas for the treatment of hyperlipidemia. J Ethnopharmacol. 2012;140(2):345-367. http://dx.doi.org/10.1016/j.jep.2012.01.027

3. Jang JY, Kim HN, Kim YR, Choi YW, Choi YH, Lee JH, et al. Hexane extract from Polygonum multiflorum attenuates glutamate-induced apoptosis in primary cultured cortical neurons. J Ethnopharmacol. 2013;145(1):261-268. http://dx.doi.org/10.1016/j.jep.2012.10.061 "tryptophan metabolism (ko00380)". It may regulate the metabolism of tryptophan to influence phenylalanine synthesis. Whether or not, THSG biosynthesis must be complicated and correlate with multiple pathways. More than just a phenylalanine pathway also must have common effect of ATP energy metabolism active and other related reactions to strengthen or weaken the process. Our research isolated several candidate genes that might participate in the THSG biosynthesis. Further research will focus on the characterization of candidate genes and gene screening to transcriptome in more diverse tissues.

4. Wang M, Zhao R, Wang W, Mao X, Yu J. Lipid regulation effects of Polygoni Multiflori Radix, its processed products and its major substances on steatosis human liver cell line L02. J Ethnopharmacol. 2012;139(1):287-293. http://dx.doi.org/10.1016/j.jep.2011.11.022

5. Lv G, Lou Z, Chen S, Gu H, Shan L. Pharmacokinetics and tissue distribution of 2,3,5,4'-tetrahydroxystilbene-2-O- $\beta$-D-glucoside from traditional Chinese medicine Polygonum multiflorum following oral administration to rats. J Ethnopharmacol. 2011;137(1):449-456. http:// dx.doi.org/10.1016/j.jep.2011.05.049

6. Zhang JK, Yang L, Meng GL, Fan J, Chen JZ, He QZ, et al. Protective effect of tetrahydroxystilbene glucoside against hydrogen peroxide-induced dysfunction and oxidative stress in osteoblastic MC3T3-E1 cells. Eur J Pharmacol. 2012;689(1-3):31-37. http://dx.doi. org/10.1016/j.ejphar.2012.05.045

7. Yao WJ, Fan WJ, Huang C, Zhong H, Chen XF, Zhang W. Proteomic analysis for anti-atherosclerotic effect of tetrahydroxystilbene glucoside in rats. Biomed Pharmacother. 2013;67(2):140-145. http://dx.doi. org/10.1016/j.biopha.2012.10.007

8. Sun FL, Zhang L, Zhang RY, Li L. Tetrahydroxystilbene glucoside protects human neuroblastoma SH-SY5Y cells against MPP+-induced cytotoxicity. Eur J Pharmacol. 2011;660(2/3):283-290. http://dx.doi. org/10.1016/j.ejphar.2011.03.046

9. Chong J, Poutaraud A, Hugueney P. Metabolism and roles of stilbenes 
in plants. Plant Sci. 2009;177(3):143-155. http://dx.doi.org/10.1016/j. plantsci.2009.05.012

10. Hartmann T. From waste products to ecochemicals: fifty years research of plant secondary metabolism. Phytochemistry. 2007;68(22-24):28312846. http://dx.doi.org/10.1016/j.phytochem.2007.09.017

11. Sheng SJ, Liu ZY, Zhao W, Shao L, Zhao SJ. Molecular analysis of a type III polyketide synthase gene in Fallopia multiflora. Biologia (Bratisl.). 2010;65(6):939-946. http://dx.doi.org/10.2478/s11756-010-0113-8

12. Shao L, Zhao SJ, Cui TB, Liu ZY, Zhao W. 2,3,5,4'-tetrahydroxystilbene2-O- $\beta$-D-glycoside biosynthesis by suspension cells cultures of Polygonum multiflorum Thunb and production enhancement by methyl jasmonate and salicylic acid. Molecules. 2012;17(12):2240-2247. http:// dx.doi.org/10.3390/molecules 17022240

13. Peng T, Zhu XF, Fan QJ, Sun PP, Liu JH. Identification and characterization of low temperature stress responsive genes in Poncirus trifoliata by suppression subtractive hybridization. Gene. 2012;492(1):220-228. http://dx.doi.org/10.1016/j.gene.2011.10.025

14. Gao M, Wang Q, Wan R, Fei Z, Wang X. Identification of genes differentially expressed in grapevine associated with resistance to Elsinoe ampelina through suppressive subtraction hybridization. Plant Physiol Biochem. 2012;58:253-268. http://dx.doi.org/10.1016/j. plaphy.2012.07.009
15. Pimentel P, Salvatierra A, Moya-León MA, Herrera R. Isolation of genes differentially expressed during development and ripening of Fragaria chiloensis fruit by suppression subtractive hybridization. J Plant Physiol. 2010;167(14):1179-1187. http://dx.doi.org/10.1016/j. jplph.2010.03.006

16. Wang YS, Gao LP, Wang ZR, Liu YJ, Sun ML, Yang DQ, et al. Lightinduced expression of genes involved in phenylpropanoid biosynthetic pathways in callus of tea [Camellia sinensis (L.) O. Kuntze]. Sci Hortic. 2012;133:72-83. http://dx.doi.org/10.1016/j.scienta.2011.10.017

17. Fukushima EO, Seki H, Ohyama K, Ono E, Umemoto N, Mizutani M, et al. CYP716A subfamily members are multifunctional oxidases in triterpenoid biosynthesis. Plant Cell Physiol. 2011;52(12):2050-2061. http://dx.doi.org/10.1093/pcp/pcr146

18. Seki H, Sawai S, Ohyama K, Mizutani M, Ohnishi T, Sudo H, et al. Triterpene functional genomics in licorice for identification of CYP72A154 involved in the biosynthesis of glycyrrhizin. Plant Cell. 2011;23(11):4112-4123. http://dx.doi.org/10.1105/tpc.110.082685

19. Carelli M, Biazzi E, Panara F, Tava A, Scaramelli L, Porceddu A, et al. Medicago truncatula CYP716A12 is a multifunctional oxidase involved in the biosynthesis of hemolytic saponins. Plant Cell. 2011;23(8):3070-3081. http://dx.doi.org/10.1105/tpc.111.087312 